\title{
CTS-API Disease Main Type Terminology
}

National Cancer Institute

\section{Source}

National Cancer Institute. CTS-API Disease Main Type Terminology. NCI Thesaurus.

Code C138190.

Terminology subset used for the purpose of supporting the CTS-API disease main type terminology. 